\title{
Research on the Strategy of Urban Ecological Competitiveness under the Integration of the Yangtze River Delta -Taking Meilong Town of Shanghai as an example
}

\author{
Shuai $\mathrm{Li}^{1{ }^{1}}{ }^{*}$, Yan Yang ${ }^{1}$, Yiting $\mathrm{Yu}^{1}$, Li Zhao ${ }^{1}$, Linlin $\mathrm{Li}^{1}$ \\ ${ }^{1}$ College of Urban Construction and Safety Engineering, Shanghai Institute of Technology, Shanghai, 201418, China
}

\begin{abstract}
As the main form of new urbanization, urban agglomeration regional integration has gradually become an important carrier and platform for leading China's economic transformation and upgrading. The Yangtze River Delta urban agglomeration is one of the largest urban agglomerations in China, and its integrated and coordinated development is of great significance to China. Taking the Meilong Town of Shanghai as an example, this paper summarizes the impact and role of high-quality urban development under the integration of the Yangtze River Delta. Through the analysis from the perspectives of spatial structure, land use layout, urban renewal, and water grid bureau, the urban development under the integration of the Yangtze River Delta was initially explored.
\end{abstract}

\section{Yangtze River Delta Integration Strategy}

The rise of the Yangtze River Delta integration into a national strategy will reshape the new pattern of Shanghai's development. Since 2018, the integration of the Yangtze River Delta has been significantly accelerated, and a long-term cooperation and exchange mechanism has been gradually established. Li Qiang, secretary of the Shanghai Municipal Party Committee, pointed out that the integration of the Yangtze River Delta has become a national strategy, which is not only a common opportunity for the three provinces and one city, but also our common responsibility. First, the Yangtze River Delta Integrated Demonstration Zone will lead the Yangtze River Delta to build a more open and coordinated regional spatial pattern. Second, the Yangtze River Delta integration strategy will comprehensively promote the high-quality development of urban space in Shanghai, and promote the transformation and upgrading of urban and rural areas such as Meilong Town in Shanghai to achieve leap-forward development.

\section{Meilong Town Overview}

Meilong Town is located in the east of Minhang District of Shanghai. It is connected to Huaying Town, Lingyun Street and Kangjian Street in Xuhui District in the east, Wusong Town in the south, Xinzhuang Town and Yanqiao Town in the west, and Hongqiao Town and Gumei Street in the north. There are Xujiahui, Qiantan, Xinzhuang and other urban centers. The area is 28.07 square kilometers and it has 15 administrative villages, 4 reformed villages and 62 neighborhood committees.
At present, Meilong Town has become an important living area of Minhang District, an important area for economic transformation and upgrading, a regional business center and a green ecological town.

\section{Based on the overall positioning of Meilong Town under the integration of the Yangtze River Delta}

The overall orientation of urban space development under the background of the integration of the Yangtze River Delta is reflected in a total of three points, including overall positioning and development strategies. The development and orientation of urban space under the background of the integration of the Yangtze River Delta should be consistent with the future industrial, social, environmental and cultural development of Meilong Town.

Overall positioning: three generations of integration, innovative home.This positioning contains the following connotations: The first is spatial integration. Production and urban, urban and rural, urban and natural ecological environment should be organically integrated and support each other. Second is the innovation ecology. It is necessary to establish a multi-dimensional, networklike, small-scale, high-density innovative enterprise gathering space. The third is cultural heritage. Taking the history and culture of Meijiagan as the direction and inheritance, we will focus on the protection, development and utilization of Meijialong culture, continue the historical context, and build a cultural Meilong. The fourth is the Hewan Ancient Road. Taking the historical allusions of Caojiaqiao and Chunshentang as the starting point, explore the urban spatial

*Corresponding author: 693483030@qq.com 
connotation of the unique Hewan ancient road in Meilong, create a good ecological and livable environment in the surrounding area, and create a city card with Meilu characteristics, relying on the hometown of Meijia. The inner requirements of the Hewan Ancient Road shape the Meilong culture and the Meilong spirit.

\section{Space conception and urban construction}

\subsection{Urban spatial structure}

\subsubsection{Status of urban spatial structure}

From the ecological skeleton: the ecological corridor is basically formed, but lacks open space, and has poor connection with the open space landscape and sight of Pujiang; the ecosystem is not systematic.

From the residential group: the residential group is more dispersed, and there is still a big gap between the positioning of the livable community, and the proportion of residential land planning in the adjacent area of Binjiang is low.

From the industrial distribution: through the existing planning, Meixi Town's current industrial storage transformation area of 592 hectares, most of which involves the secondary development of stock land. The difference in the output value of unit land is large, the number of migrants is large, the proportion of secondgeneration personnel is large, and the adjustment of scale structure is difficult.

\subsubsection{The nature of the town and the overall goal}

The nature of the town: a historical and cultural town featuring the ancient towns of the water town and the science and technology. The suburbs of Shanghai have the function of the international main city----the famous town of Jiangnan Water Town and the charming town of Science and Technology.

The overall goal: to highlight the characteristics of the famous cultural towns in the south of the Yangtze River; to strengthen the construction of manufacturing brands, to create a functional composite innovation and entrepreneurship practice zone.

\subsubsection{Spatial layout principles}

First of all, we should establish an ecological red line management system and strictly follow the ecological security bottom line. Secondly, we should strengthen the bottom line constraints and green leadership of the ecological environment and continue to promote green transformation and development in the fields of energy, industry, transportation and construction.

Second, highlight the traffic skeleton guidance. Meilong Town has initially formed a prototype of "three vertical and four horizontal". The next step is to strengthen the compact layout of urban spatial structure, strengthen the structural model of "three vertical and four horizontal", and develop multi-level, networked and multi-functional towns. Space skeleton system.

\subsection{Land use layout}

Guide the intensive use of land. Further improve the land use efficiency within the land development boundary, moderately increase the intensity of residential land, and ensure the supply of future strategic emerging industrial land.Strengthen the transition from secondary production to tertiary production. According to the current regional industrial distribution and production efficiency, accelerate the upgrading of labor-intensive industrial enterprises and accelerate the transformation from traditional secondary roduction to tertiary production. Enhance functional integration. According to the new round of overall planning and positioning of Minhang District, Meilong Town mainly focuses on residential functions. In the future, under the premise of ensuring the overall planning of the district, the industrial park will be integrated with the development of the living and service space, encourage employment in the community, improve public services, and promote the integration of life, employment and leisure.

Promote mixed use of land.Exploring the comprehensive development of commercial, office, residential, public services and municipal facilities, and encouraging public service facilities, industrial land, and storage land to further improve land use quality and efficiency according to the needs of the industry, mixed land development and composite utilization of buildings.

\subsection{Urban Organic Update}

\subsubsection{Main types and problems of urban renewal in Meilong Town}

\section{(1) Urban-rural integration}

With the urbanization process in Shanghai, the development of real estate industry and the construction of industrial parks in Meilong Town accelerated, and collective land gradually changed into construction land, but some original villages were still retained. Later, the gathering of outsiders caused the villages in the city to be mixed with the modern cities, resulting in problems such as safety, environment and sanitation. Meilong Town is a town in the outer ring of the outer ring of Meilong Town.

There are the following problems in the villages of the city:

First, the house is in disrepair and the people are intensive.Second, the architectural style problem is outstanding and the rectification is more difficult.Third, the personnel structure is complex and internal management is difficult. 


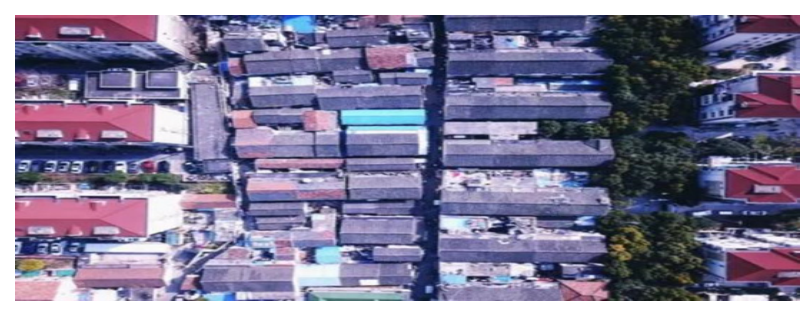

Fig. 1. Single and double village

\section{(2) industrial area}

Most of the old industrial areas in Meilong Town are machinery factories and textile factories. Replacement, relocation, inefficient idle land and building utilization have become the main breakthroughs in the organic renewal of Meilong Town. According to the situation of existing industrial enterprises, it can be divided into large-scale industrial plants and space-distributed lowlevel industrial plants. The Asia-Pacific brewery, which is idle in Yimei Road, has a well-preserved architectural form, adjacent to the river, opposite to the residential area, and has good conditions for transformation and development. It can replace some building functions in the plant area, improve the environment, and increase public green space or public space along the road. Demolition of the enclosed wall to improve the city's appearance.

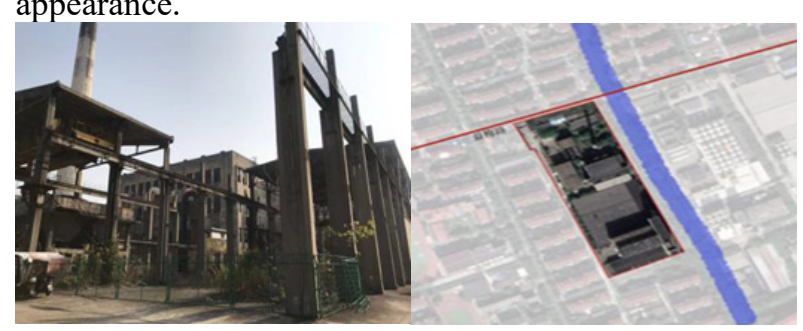

Fig. 2. Yimei Road Asia Pacific Brewery

The industrial plants along the river in Hongmei South Road-Dianpu River section are mostly concentrated in traditional industries such as mining industry and raw material industry; the production products are single, the processing level is low, mainly producing raw materials, primary products and some intermediate products, and the land intensive utilization rate not tall.

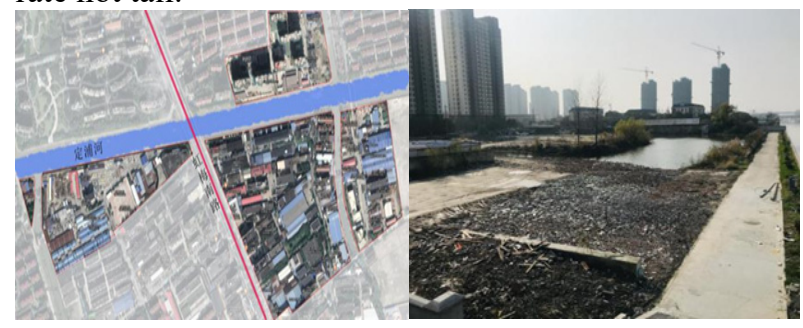

Fig. 3. Industrial area on both sides of the Dianpu River

\subsubsection{Evolution and Causes of Urban Renewal in Meilong Town}

In the late $1950 \mathrm{~s}$, Meilong Town set up a social industrial base in the establishment of a social industrial base. The urban renewal of Meilong began after the reform and opening up, mainly through the transformation of the old town in the 1980s, the demolition of the old street and the construction of the new street; after the 1990s, the large-scale old districts were rehabilitated. In the first decade of the 21 st century, with the rise of the real estate industry, the transformation of the old districts in Meilong Town and surrounding areas accelerated. The urban renewal of Meilong Town is mainly based on the renovation of old districts and the transformation of industrial plants, which is basically consistent with the situation in other parts of Shanghai.

\subsubsection{Meilong Town Organic Renewal Strategy}

(1)General principle

The urban organic renewal of Meilong Town should adhere to the principles of respecting the law, planning first, coordinating time series, and classifying guidance.

(2)Urban-rural integration

Focus on people's livelihood and reform in batches. Structural optimization, facility first function replacement, and environmental improvement.

(3)Industrial land renewal

Industrial upgrading and efficient land use. Functional replacement, cultural introduction. Urban community, mixed function.

\subsection{Water Town Charm Charming Town}

\subsubsection{Water network space pattern}

(1) The current situation of water network spatial pattern in Meilong Town

Advantages: The water system in the town is sufficient, laying the foundation for the construction of the water town pattern. The three main water systems pass through the township from west to east, bringing sufficient water and landscape resources to the township.

Disadvantages: The water network system is dense, but it has not been sorted out. The water form shows a mixed state of natural and artificial forms. Many water systems have broken ends at the end and fail to form a continuous water network system.

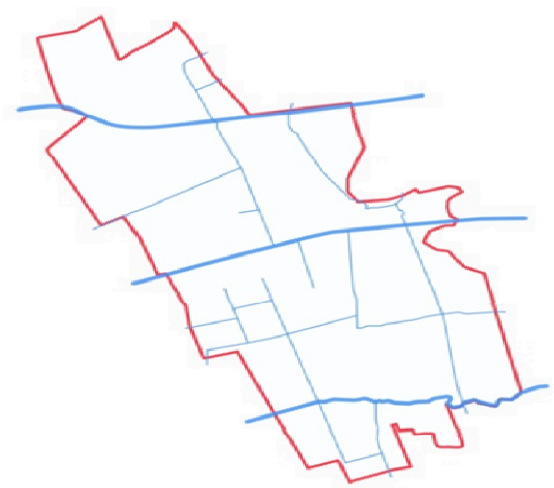

Fig.4. Water system map 
(2) Water Grid Bureau to create goals

Goal: According to the spatial pattern of the water, the water source integrates into life.

Characteristics of the water system: Sanhe Ming wears through the city, and the multi-stream is dark and the water network is reflected.Sanhe refers to Dianpu River, Chunshentang and Songiiahe. Make full use of the rich features of the water system, the water network densely intersected and criss-crossed to achieve spatial changes.

(3) Water Grid Bureau Planning

1) Reproduce the river and perfect the water network.

2) Protect the water body and control the edges.

3) Retreat to the river and coordinate the style.

4) Classification of shorelines and diversity of waterfront.

\subsection{2 texture characteristics of the neighbourhood}

(1) Summary of the current situation of the texture of the neighborhood.

The texture of the street is separated and the waterfront activity is insufficient. First of all, Meixi Town's neighborhood features a historical origin. The industrialization and the rapid development of the city have caused the street human scale to gradually disappear, and the industrial legacy is too heavy. Jacobs pointed out in "The Life and Death of American Big Cities" that the emphasis on modern transportation and functional zoning and large-scale independent single buildings undermines the traditional cultural context of the city. The large enclosed residential area in the area transforms the traditional and vibrant streets into motor vehicle passages. The obstacles to the passage of the roads make the large residential communities in contemporary cities become urban isolated islands, leading to the disappearance of urban vitality. At present, the texture of architecture and street space is summarized from the whole town level. Secondly, the waterfront space is the regional vitality center. As the transportation mode changes from land and water transportation to land and land transportation, the waterfront space gradually withdraws from the living center. Many of the waterfront spaces in Meilong Town have been squeezed by industrial land, and they have lost accessibility due to poor accessibility and lack of high-quality public space.

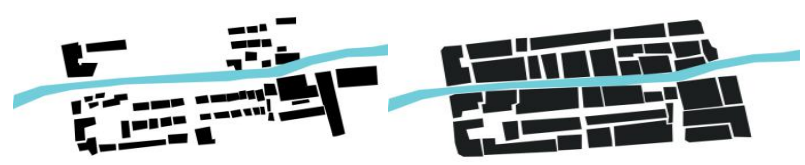

Fig.5. Cao Xingcun's architectural texture and its street texture
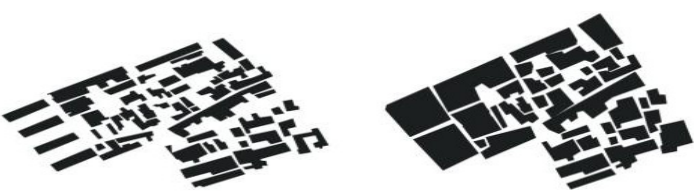

Fig.6. Zhu Jiaxin Village Architectural Texture and Its Street Texture

(2) Extraction of traditional elements of Meilong Town's texture

Street texture. Street and street space can best reflect the characteristics and forms of Jiangnan style. The characteristics of Jiangnan city water towns also reflect the parallel urban spatial structure of waterways. Streetbuilding-river formation is interdependent, closely related, self-organizing spatial layout.
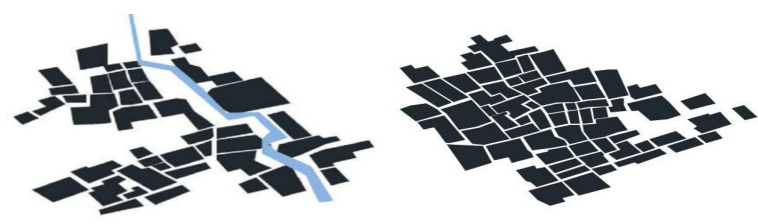

Fig.7. Traditional street texture

Water falls. Its main features are that the street scale is small, the road network density is high, the twists and turns, the primary and secondary roads are not obvious, and the main roads are adjacent to water. The building texture is mainly reflected in the courtyard relationship and the road connection. The building has a deeper depth, a compact layout and a higher density.
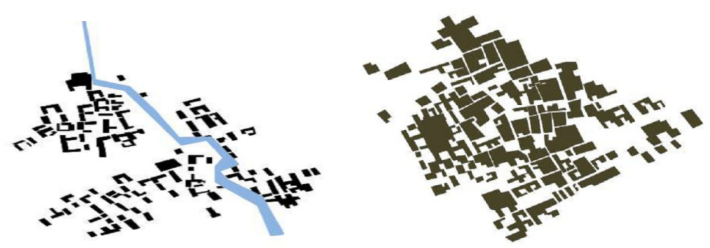

Fig.8. Traditional water texture

Town area space. The traditional township is mainly located in the northern part of Meilong Town, which is a mixture of traditional villages and built-up areas. The primary and secondary roads in the area are clearly graded, the roads in the residential area are slightly twisted, the scale of the block is large, the size of the building is not uniform, and the construction period has been lacking in planning in recent decades. The new town area is located in the middle, and the road is mainly embodied in the form of square grid. The primary and secondary roads are obvious, and the scale of the block is large. The building form is relatively regular, the density is moderate, and there is more opening space. 

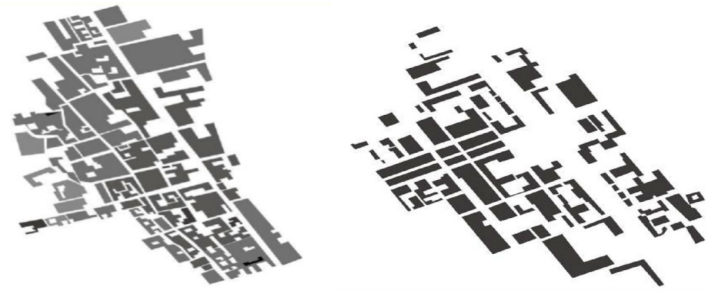

Fig.9. Town area texture

(3) Meilong Town's neighborhood texture construction

Different classification methods and design features are adopted for different functional division characteristics of cities and towns, including urban traditional construction style area, town center style area, traditional village style area, and waterfront space style area.

1) Urban traditional construction style area

It mainly includes urban history and culture, traditional business services, tourism services, business services, industrial parks and supporting residential sectors. On the planning scale, it inherits the characteristics of traditional Jiangnan cultural towns.

Construction measures: intensive land use, compact development, delineating urban growth boundaries; establishing guidelines for urban construction layout, determining the layout and types of roads and lanes, public open spaces, etc. according to the style and division; creating a dynamic environment and diversifying street life Experience.

2) Town Center Center Scenic Area

The innovative modern town that combines the traditional elements of Jiangnan is mainly located in the middle of Jindu Road, the living service, commercial and trade service area and the surrounding radiation area. The style features are located in the modern development zone of the town.

Construction easures: compact integration, comprehensive development of land functions; advocacy of multi-layer high-density, local high-rise control regional centers, with the integration of traditional Jiangnan architectural courtyard layout intentions with modern features; straight roads and streets, meeting modern business functions and evacuation requirements A functional structure that combines multiple functions of living, business, culture, and leisure.

3) Traditional village style area

The village features traditional Jiangnan features, and the style is positioned as a traditional village living area. It is mainly located in the southern part of the township, combining some of the primary industries to optimize the layout of the village.

Construction measures: intensive development, demarcation of village growth boundaries; establishment of village construction guidelines, inheriting the color and volume of Jiangnan buildings, controlling the height of buildings 1 to 3, prohibiting the emergence of largescale buildings with special features; retaining features or use intact The village house has been remodeled to give new functions;

4) Waterfront vitality area
First of all, we should set up ecological revetment and hydrophilic facilities at the core waterfront space nodes to enhance the accessibility of waterfront space. Secondly, we should set up an ecological revetment at the waterfront space node to create a hydrophilic space.

In the waterfront space where the buildings are concentrated, especially when the waterfront interface is a waterfront building and a laneway, a waterfront public corridor can be added to the outside of the building to enhance the accessibility of the waterfront. Recreational and fitness facilities should be set up in the shore green belt for residents to enjoy.

\section{References}

1. Liu Shilin. The globalization process of urban agglomerations and China's experience [J]. Academics, 2012 (06).

2. $\mathrm{Xu}$ Qin. The development of the Yangtze River Delta under the multi-center structure [J]. Modern Economic Research, 2018 (09)

3. Luigi T. Zhu Yingming. Study on the Regional Structure Characteristics and Development Trend of Urban Agglomerations in China[J]. Urban Planning Journal, 2001, (7): 55-57)

4. QianHuixiong. Measurement of Regional Economic Integration in the Yangtze River Delta Region [J]. Finance and Trade Research, 2010(5)

5. Ren Zhenzhu. The Future of the Yangtze River Delta: Opportunities and Challenges [M]. People's Publishing House, August 2011 